\section{Chylothorax as a complication of oesophageal sclerotherapy}

\author{
Scott D Nygaard, Herbert A Berger, \\ Robert B Fick
}

\begin{abstract}
Chylothorax is an unusual complication of sclerotherapy for oesophageal varices. A patient is described in whom a massive chylous effusion followed sclerotherapy with repeated injections of $1.5 \%$ sodium tetradecyl sulphate. The thoracic duct traverses the posterior mediastinum in close proximity to the oesophagus, and may be disrupted by injections at mid oesophageal level.
\end{abstract}

Disruption of the thoracic duct in the mediastinum may allow chyle to enter the pleural space. These chylous pleural effusions contain a triglyceride concentration greater than $1 \cdot 1$ $\mathrm{g} / \mathrm{l}$ and chylomicrons on lipoprotein analysis. This disorder may be caused by penetrating chest trauma, hyperextension of the spine, or iatrogenic injuries resulting from cardiovascular surgery. ${ }^{1}$ Chylothorax may also occur with damage to the thoracic duct from neoplasia, filariasis, and lymphangitis. The thoracic duct runs posterior to the middle third of the oesophagus, where it may be damaged during oesophageal sclerotherapy.

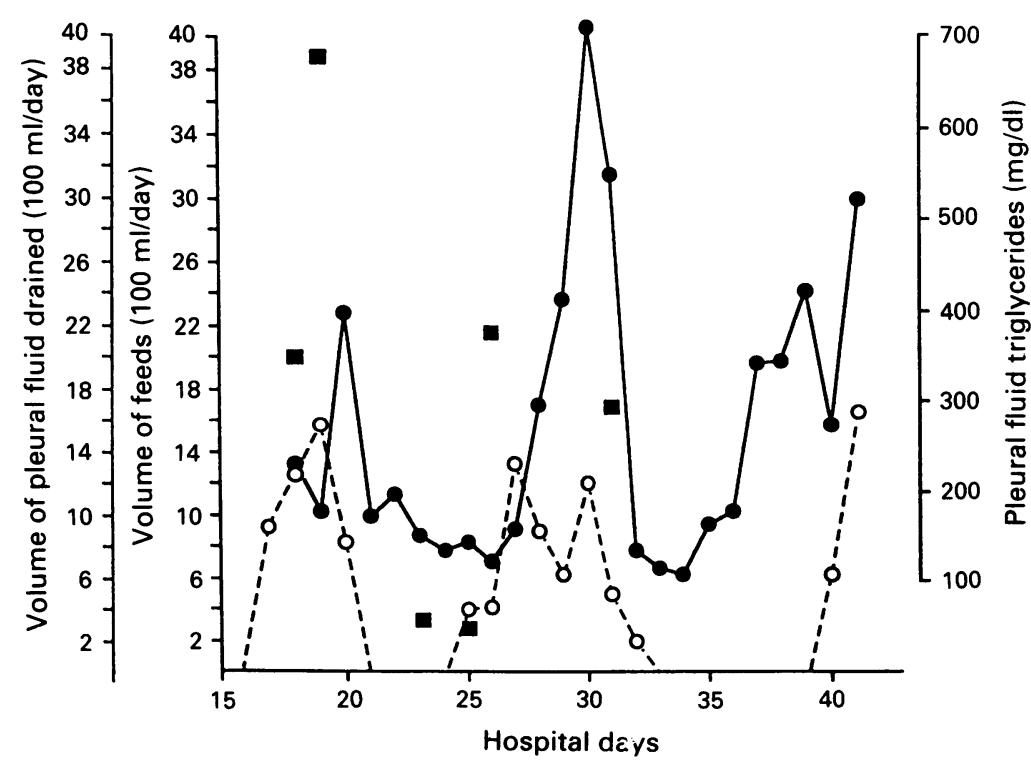

Hospital course charted as days from admission (sclerotherapy performed on hospital days 2, 5, 6, and 11; enteral feeding on day 17), showing how the volume of pleural fluid drained ( ) closely mirrored the volume of the enteral feeds infused $(\mathrm{O}--\mathrm{O})$; pleural fluid triglyceride concentration ( ) fell (days 23 and 25 ) when enteral feeding was discontinued. $1 \mathrm{mg}$ triglyceride $/ \mathrm{dl}=0.01129 \mathrm{mmol} / \mathrm{l}$.
We report a case in which this appears to have occurred.

\section{Case report}

A 56 year old woman with a history of chronic active non-A, non-B viral hepatitis was admitted to the University of Iowa Hospitals and Clinics for management of bleeding oesophageal varices. Placement of a SengstakenBlakemore tube and a vasopressin infusion at 0.4 units $/ \mathrm{min}$ controlled bleeding initially. Further episodes of variceal bleeding occurred, however, in the mid and distal oesophagus two, five, and six days after admission and sclerotherapy with $1.5 \%$ sodium tetradecyl sulphate was required. Follow up endoscopy showed several small $(<0.5 \mathrm{~cm})$ oesophageal ulcerations. On the 11th day in hospital the patient passed maroon coloured stools. Endoscopy showed a large bleeding varix on the posterior wall of the oesophagus. Two intravariceal injections of sclerosant failed to control bleeding so a paravariceal injection was attempted, without success. A final unsuccessful attempt was made, with six radial injections of $1 \mathrm{ml}$ of sclerosant at the gastro-oesophageal junction. Subsequently placement of a Minnesota tube and vasopressin infusion stopped the bleeding. On the 12th hospital day an emergency LaVeen shunt and mesocaval shunt procedure was carried out.

Intravenous hyperalimentation was given until enteral tube feeding was initiated on the fifth postoperative day (the 17th hospital day). One day after starting enteral feeding the patient became breathless and a chest radiograph showed a large right pleural effusion. Thoracocentesis yielded reddish yellow turbid fluid without evidence of infection but with a raised triglyceride concentration of $350 \mathrm{mg} / \mathrm{dl}$ (3.95 $\mathrm{mmol} / \mathrm{l})$; the simultaneous serum triglyceride concentration was $70 \mathrm{mg} / \mathrm{dl}(0.79$ $\mathrm{mmol} / \mathrm{l})$. A chest tube drained copious amounts of cream coloured fluid resembling the enteral tube feedings. Methylene blue was added to the tube feed but was not found in the pleural fluid.

On the patient's 21 st day in hospital enteral tube feedings were discontinued and intravenous hyperalimentation was restarted (figure). Over the next few days the chylous effusion became amber coloured and the volume that was drained fell. On the 24th hospital day subcutaneous isosulphate blue ( $1 \mathrm{ml} 1 \%$ solution) injection confirmed the decreased extravasation of thoracic fluid into the pleural space. The fluid subsequently drained from the chest tube and remained clear of dye.

On her 25th day in hospital the patient was given a high fat enteral tube feeding challenge. Although initially non-chylous chest tube drainage increased as enteral tube feeding continued, it eventually decreased only when intravenous nutritional support was restarted. The patient refused further surgical intervention.

On her 32nd day in hospital peritoneal fluid and blood cultures grew Eschericia coli. The 
next day she produced a maroon coloured stool, her condition continued to decline, and she died on her 41st day in hospital. Necropsy was refused.

\section{Discussion}

Sclerotherapy for bleeding oesophageal varices was first described in 1939, but has been used widely only since 1979 . It has been shown to reduce the risk of bleeding and improve the chances of survival. ${ }^{2}$ Several different sclerosing agents have been used, including sodium morrhuate, ethanolamine oleate, and sodium tetradecyl sulphate. All have been associated with complications (table).

The development of a chylothorax 16 days after the start of sclerotherapy is likely to have resulted from either inadvertent direct disruption of the thoracic duct or from localised mediastinitis disrupting the thoracic duct. The only publication we found is a surgical report of chylothorax complicating sclerotherapy that attributed the sudden and massive pleural effusion to instrumental perforation in a patient with cirrhosis. Complications are more likely in the presence of cirrhosis because of the close contact of a dilated thoracic duct with the oesophagus and right pleural cavity. ${ }^{6}$

The pleural effusion developed within 24 hours of the start of enteral feeding. This would be expected to fill the thoracic duct with triglycerides, and each time enteral feeding was discontinued the fluid drained from the pleural space decreased substantially. The continued high output of chyle from the chest tube suggests that a fistula had developed between the thoracic duct and the right pleural cavity.

Reported complications of sclerotherapy

Atelectasis $^{3}$

Aspiration pneumonia ${ }^{2}$

Adult respiratory distress syndrome ${ }^{2}$

Pleural effusion ${ }^{3}$

Oesophageal-pleural fistula ${ }^{2}$

Bronchoesophageal fistula ${ }^{2}$

Mediastinitis $^{4}$

Pneumothorax

Chylothorax (and this report)
The continued output of chyle results in malnutrition and immunological compromise. These factors are likely to have contributed to the development of infection and possibly to the patient's death. Patients with chylothorax become malnourished and their immunity is compromised because of the fat, protein, and lymphocytes lost in the drained pleural fluid. ${ }^{7}$ The mortality for chylothorax ranges from $30 \%$ to $50 \%$. Treatment is aimed at maintaining the patient's nutritional state, reducing the flow of thoracic duct lymph, and expanding the affected lung.

A disrupted thoracic duct may close spontaneously within a week of conservative treatment. Treatment to reduce production of chyle includes bed rest and total parenteral nutrition $^{2}$ or a high protein enteral diet with medium chain triglycerides replacing fat. Drainage of pleural fluid for more than seven days requires aggressive treatment to avoid further complications. Management options include pleurodesis and ligation of the thoracic duct. Because of the many lymphatic and lymphaticovenous anastomoses, thoracic duct ligation has few side effects. ${ }^{8}$ Physicians should be aware that patients receiving mid oesophageal sclerotherapy may develop a chylothorax.

1 Fairfax AJ, McNabb WR, Spiro SG. Chylothorax: a review of 18 cases. Thorax 1986;41:880-5.

2 Schuman B, Beckman J, Tedesco F, Griffin J, Assad R. Complications of endoscopic injection sclerotherapy. A review. Am J Gastroenterol 1987;82:823-30.

3 Saks B, Kilby A, Dietrich P, Coffin L, Krawitt E. Pleural and mediastinal changes following endoscopic injection sclerotherapy of oesophageal varices. Radiology 1983;149: 639-42.

4 Ayres SJ, Goff TS, Warren GH. Endoscopic sclerotherapy for bleeding oesophageal varices: Effects and complications. Ann Intern Med 1983;98:900-3.

5 Gertach P, Mosimann R. Chylothorax complicating sclerotherapy for oesophageal varices. Br J Surg 1983; 70:562.

6 Dumont AE, Witte MH. Significance of excess lymph flow in the thoracic duct in patients with hepatic cirrhosis. $A m \mathrm{~J}$ Surg 1966;112:401-6.

7 Machleder HI, Paulus H. Clinical and immunological alterations observed in patients undergoing long-term thoracic duct drainage. Surgery 1978;84:157-65.

8 Williams KR, Burford TH. The management of chylothorax. Ann Surg 1964;160:131-40. 